\title{
Radio-frequency sheath voltages and slow wave electric field spatial structure
}

Laurent Colas', Ling-Feng Lu, Alena Křivská, and Jonathan Jacquot

Citation: AIP Conference Proceedings 1689, 050009 (2015); doi: 10.1063/1.4936497

View online: http://dx.doi.org/10.1063/1.4936497

View Table of Contents: http://aip.scitation.org/toc/apc/1689/1

Published by the American Institute of Physics 


\title{
Radio-Frequency Sheath Voltages and Slow Wave Electric Field Spatial Structure.
}

\author{
Laurent Colas ${ }^{1, a}$, Ling-Feng Lu ${ }^{1}$, Alena Křivská ${ }^{2} \&$ Jonathan Jacquot $^{3}$ \\ ${ }^{1}$ CEA, IRFM, F-13108 Saint-Paul-Lez-Durance, France. \\ ${ }^{2}$ LPP-ERM-KMS, TEC partner, Brussels, Belgium. \\ ${ }^{3}$ Max-Planck-Institut für Plasmaphysik, Garching, Germany. \\ ${ }^{\text {a) Corresponding author: laurent.colas@,cea.fr }}$
}

\begin{abstract}
We investigate theoretically how sheath radio-frequency (RF) oscillations relate to the spatial structure of the RF parallel electric field emitted by Ion Cyclotron (IC) wave launchers, using a simple model of Slow Wave (SW) evanescence coupled with Direct Current (DC) plasma biasing via sheath boundary conditions in a plasma-filled 2dimensional (parallel, radial) rectangle. Within a "wide sheaths" asymptotic regime, valid for large-amplitude near RF fields, our model becomes partly linear: the sheath oscillating voltage at open field line boundaries is a linear combination of elementary contributions by every source point of the radiated RF field map. These individual contributions are all the more intense as the SW emission point is toroidally nearer to the sheath walls. A limit formula is given for a source infinitely close to the sheaths. The decay of sheath RF voltages with the sheath/source parallel distance is quantified as a function of two characteristic SW evanescence lengths. Decay lengths are smaller than antenna parallel extensions. The sheath RF voltages at an IC antenna side limiter are therefore mainly sensitive to SW emission near this limiter, as recent observations suggest. Toroidal proximity effects could also explain why sheath oscillations persist with antisymmetric strap toroidal phasing, despite the parallel anti-symmetry of the radiated field map. They could also justify current attempts at reducing the RF fields induced near antenna boxes to attenuate sheath oscillations in their vicinity.
\end{abstract}

\section{EXPERIMENTAL INDICATIONS OF LOCALIZED RF-SHEATH EXCITATION}

In magnetic fusion devices, non-linear wave-plasma interactions in the Scrape-Off Layer (SOL) often set operational limits for Radio-Frequency (RF) heating systems via impurity production or excessive heat loads. Peripheral Ion Cyclotron (IC) power losses are generally attributed to RF sheath rectification. How this non-linear process depends on the geometry and electrical settings of the IC wave launchers remains largely unknown, despite crucial technological implications. In realistic geometry over the scale length of an IC antenna, rectification is often modelled in analogy with a double Langmuir probe driven by an oscillating voltage $\tilde{V}$, estimated as the field-lineintegrated RF field, $E_{/ /}$parallel to the confinement magnetic field $\mathbf{B}_{0}[1] . \tilde{V}=\int E_{/ / .} d l$ is generally evaluated from RF field simulations without sheaths. This simple approach was challenged though. In a series of Tore Supra (TS) experiments, the left-right ratio of IC strap voltage amplitudes was varied by different means [2]. Over this scan, the antenna side limiter near the strap with higher voltage heated up, while the remote limiter cooled down. A similar dissymetrization on ASDEX-Upgrade produced opposite variations of shunt RF currents at two opposite antenna limiters [3]. These trends can hardly be explained using a single parameter relevant all along an open field line, $\tilde{V}$ or any other one. Beyond line-integrated quantities, these observations suggest that the toroidal distance between radiating elements and passive walls might play a role in the RF-sheath excitation. By coupling RF wave propagation and Direct Current (DC) plasma biasing via RF and DC sheath boundary conditions closer to the first principles, this contribution tries to reconcile modelling and experiments. Within restrictive assumptions on geometry, wave amplitude and polarization, we investigate theoretically how sheath RF oscillations relate to the spatial structure of the RF electric fields $E_{/ / a p}$ emitted by the wave launchers, looking for parallel proximity effects. Concrete implications for present and future IC antennas are discussed, as well as limitations of the proposed model. 


\section{COUPLED SLOW WAVE PROPAGATION AND DC PLASMA BIASING BY RADIO- FREQUENCY (RF) SHEATHS: OUTLINE OF AN ASYMPTOTIC MODEL}

Our model of coupled RF wave propagation and DC plasma biasing, SSWICH-SW, was detailed in [4], [5] and is summarized here. The simulation domain features a collection of open magnetic flux tubes in the SOL plasma. It contains protruding material objects, e.g. IC antenna side limiters, intercepting the magnetic field lines and developing sheaths. These boundaries are either parallel or normal to the confinement magnetic field $\mathbf{B}_{\mathbf{0}}$. This leaves room for versatile geometries with gradients of the plasma parameters (see e.g. [5]). To ease analytical calculations, we restrict here the formalism to a 2-dimensional (2D) rectangular domain of dimensions $L_{/ /}$in the parallel direction $z$ and $L_{\star}$ in the radial direction $x$ of a flattened tokamak filled with homogeneous cold magnetized plasma. In this simple domain, sketched in gray on Fig. 1, the simulation process couples 3 steps self-consistently.

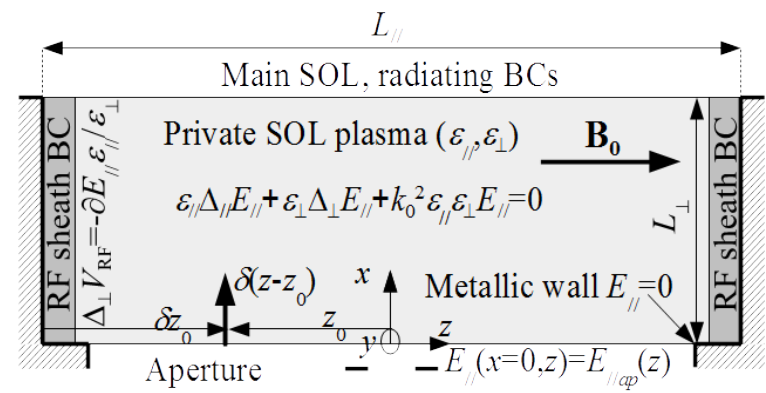

FIGURE 1: sketch of simulation domain (not to scale). Main equations and notations used in the paper
Step 1: Slow Wave propagation. The physical system is excited by a toroidal map of the parallel RF electric field $E_{/ / \text {ap }}(z)$, radiated by an IC antenna and prescribed at the outer radial boundary $x=0$ of the simulation domain. A time-harmonic Slow magnetosonic Wave (SW) with pulsation $\omega_{0}$ propagates from there according to the cold $\mathrm{SW}$ equation

$$
\varepsilon_{\perp} \Delta_{\perp} E_{/ /}+\varepsilon_{/ /} \Delta_{/ /} E_{/ /}+k_{0}^{2} \varepsilon_{/ /} \varepsilon_{\perp} E_{/ /}=0
$$

With $\Delta_{\Delta}=\partial_{\mathrm{xx}}{ }^{2} \cdot-k_{y}^{2}, \Delta_{u}=\partial_{\mathrm{zz}}{ }^{2} \cdot, \quad k_{0}=\omega_{0} / c$ the vacuum wavenumber, $\left(\varepsilon_{/ /,} \varepsilon_{1}\right)$ the diagonal elements of the cold plasma dielectric tensor [6] and $k_{y}$ a wavevector in the ignorable (poloidal) direction $y$. Equation (1) is subject

to radiating conditions at the inner radial boundary $x=L_{\star}$, as well as RF sheath boundary conditions (RF SBCs) at the parallel boundaries $z= \pm L_{/ / /}$. RF SBCs were first proposed in [7] and will be discussed below.

Step 2: RF oscillations of sheath voltage. When reaching the extremities of the open magnetic field lines, the SW fields $E_{/ /}$generate oscillations $V_{R F}$ of the sheath voltage at the RF frequency. The definition $\mathbf{E}_{\perp}= \pm \nabla_{\perp} V_{R F}$ at the sheath/plasma interface, together with the relation $\nabla .(\varepsilon \mathbf{E})=0$ valid all over the plasma, yield a diffusion equation for the sheath oscillating voltages $V_{R F}$ along the boundaries normal to $\mathbf{B}_{\mathbf{0}}$, including a source term due to the $\mathrm{SW}$,

$$
\varepsilon_{\perp} \Delta_{\perp} V_{R F}=\mp \varepsilon_{/ /} \partial_{/ /} E_{/ /}\left(x, \pm L_{/ /} / 2\right) ; V_{R F}\left(0, \pm L_{/ /} / 2\right)=V_{R F}\left(L_{\perp}, \pm L_{/ /} / 2\right)=0(2)
$$

Step 3: Rectification of the sheath oscillations. Due to the non-linear I-V characteristics of the sheath conduction current, the RF oscillations of the sheath voltage are rectified into enhanced DC biasing of the SOL plasma. Several DC biasing models exist in the literature. These will not be detailed here, but the DC plasma potential $V_{D C}$ is an increasing function of the RF voltage amplitudes $\left|V_{R F}\right|$. The DC voltage drop across the sheaths affects their width via the Child Langmuir law, and consequently their RF capacitance and the RF SBCs applied for $E_{/ /}$[7]. Therefore steps 1-3 generally need to be iterated till convergence is reached [5]. However for sheaths wider than a characteristic value, the RF SBCs were found nearly independent of the sheath widths [4] [8]. For $\mathbf{B}_{\mathbf{0}}$ normal to the wall the asymptotic RF SBCs simplify into $E_{/ /}=0$. In realistic Tore Supra simulations with self-consistent sheath widths, the near RF fields were intense enough to approach this "wide sheath" asymptotic regime [5].

\section{PARALLEL PROXIMITY EFFECTS ON THE EXCITATION OF SHEATH RF VOLTAGES BY EVANESCENT SLOW WAVES IN THE "WIDE SHEATH" REGIME}

If sheath widths are large (or alternatively if they are prescribed [9]), steps 1-3 are successive rather than coupled self-consistently. Besides, while step 3 is always non-linear, eq. (1) and (2) are linear, together with their BCs. Whatever the simulation domain, the superposition principle thus allows expressing $V_{R F}$ at any sheath boundary as the linear combination of elementary contributions by every emitting point in the input RF field map.

For the 2D rectangular domain envisaged in this paper: $V_{R F}\left(x, \pm L_{/ /} / 2\right)=\int_{-L_{/ /} / 2}^{L_{/ /} / 2} E_{/ / a p}\left(z_{0}\right) G\left(x, \mp z_{0}\right) d z_{0}$

Relation (3) looks like the integral $\tilde{V}=\int E_{/ /} d l$ used previously, with major differences however. $\left.1^{\circ}\right) V_{R F}$ relates to one sheath, whereas $\tilde{V}$ was applied between two electrodes. Depending on the parallel symmetry of the input RF field map, the two extremities of the same field line can now oscillate differently. $2^{\circ}$ ) Parallel integration is 
performed over the outer radial boundary $x=0.3^{\circ}$ ) A non-dimensional attenuation factor $G\left(x, \pm z_{0}\right)$ is applied to $E_{/ / \text {ap }}\left(z_{0}\right)$, depending both on the radial and parallel distances from the field emission point to the destination point at the sheath walls. The geometrical properties of $G\left(x, z_{0}\right)$ determine the relation of $V_{R F}$ to the SW field spatial structure.

Green's function $G\left(x, z_{0}\right)$ is the solution of equations (1) and (2) with a Dirac input field map $E_{/ / \text {ap }}(z)=\delta\left(z-z_{0}\right)$, i.e. one point source switched on in $z=z_{0}$ (see Fig. 1). For this elementary excitation, the 2D RF field pattern $E\left(x, z, z_{0}\right)$ can be determined explicitly all over the rectangular domain in the simple case $\varepsilon_{/ /}<0, \varepsilon_{\perp}<0$ (evanescent $\mathrm{SW}$ ) by the method of images. Introducing a normalization $X=x / L_{x}$ and $Z=z / L_{z}$, with characteristic evanescence lengths $L_{x}$ and $L_{z}$

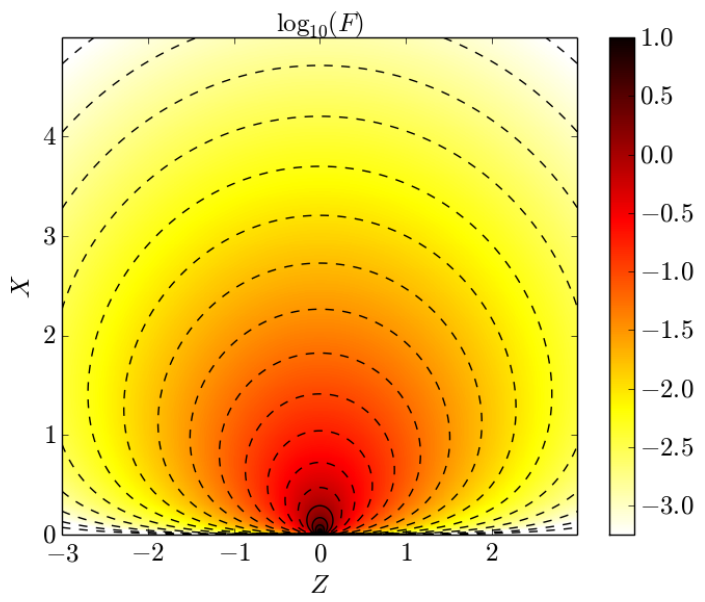

FIGURE 2. 2D plot of $F(X, Z)$ in logarithmic scale.

$$
\begin{gathered}
L_{x}=\left[k_{y}^{2}-k_{0}^{2} \varepsilon_{/ /}\right]^{-1 / 2} ; L_{z}=\left[\varepsilon_{\perp}\left(k_{y}^{2} / \varepsilon_{/ /}-k_{0}^{2}\right)\right]^{-1 / 2} \\
\text { The 2D parallel RF field pattern takes the form } \\
E\left(x, z, z_{0}\right)=L_{z}^{-1} \sum_{n=-\infty}^{+\infty}(-1)^{n} F\left(X, Z-Z_{n}\right) \\
F(X, Z)=\frac{X}{\pi R} K_{1}(R) ; z_{n}=n L_{/ /}+(-1)^{n} z_{0}
\end{gathered}
$$

In this expression $K_{1}(R)$ is a modified Bessel function of second kind and $R^{2}=X^{2}+Z^{2}$. Function $F$, plotted in 2D on Fig. 2, describes the SW evanescence from a point source in $(X, Z)=(0,0)$, in absence of parallel boundaries. For a fixed $X$ and $Z>>X>1, F$ decays as $\sim \exp (-Z)$ along the parallel direction. Study of formula (5) also reveals that $\partial_{z} E\left(x,-L_{/ /} / 2,-L_{/ /} / 2\right) \sim \varepsilon_{\perp} / \varepsilon_{/ /} \partial_{x} \delta(x)$ and $\partial_{z} E\left(x,-L_{/ /} / 2,+L_{/ /} / 2\right)=0$. Inserting (5) into equation (2), one deduces $G\left(x, \mathrm{z}_{0}\right)$ as a function of $\partial_{\mathrm{z}} E\left(x,-L_{/ /} / 2, z_{0}\right)[4]$

$$
G\left(x, z_{0}\right)=\varepsilon_{/ /}\left[k_{y} \varepsilon_{\perp} \sinh \left(k_{y} L_{\perp}\right)\right]^{-1} \int_{0}^{L_{\perp}} \partial_{z} E\left(x^{\prime},-L_{/ /} / 2, z_{0}\right) \sinh \left(k_{y} x_{\min }\right) \sinh \left(k_{y}\left(L_{\perp}-x_{\max }\right)\right) d x^{\prime}
$$

Where $x_{\min }=\min \left(x, x^{\prime}\right)$ and $x_{\max }=\max \left(x, x^{\prime}\right)$. Figure 3 plots $G\left(x, z_{0}\right)$ versus $x$ for several values of $\delta z_{0}=\left(L_{/ /} / 2+z_{0}\right)$, the parallel distance from the SW emission point $z=z_{0}$ to the left sheath boundary $z=-L_{/ / /} / 2$ (see Fig. 1). The summation in (5) extended over $|n| \leq N_{\max }$, with $N_{\max }=100$. The following parameters were used: $\varepsilon_{/ /}=-74659, \varepsilon_{\perp}=-24.31, k_{0}=0.63 \mathrm{~m}^{-1}$, $k_{y}=0, L_{/ /}=0.66 \mathrm{~m}, L_{\perp}=12 \mathrm{~mm}$. They are representative of RF-sheath simulations using realistic ASDEX-Upgrade conditions, where $\left|V_{R F}\right|$ was correlated to $\left|E_{/ / a p}\right|$ averaged over the toroidal extremities of the input field map [10].

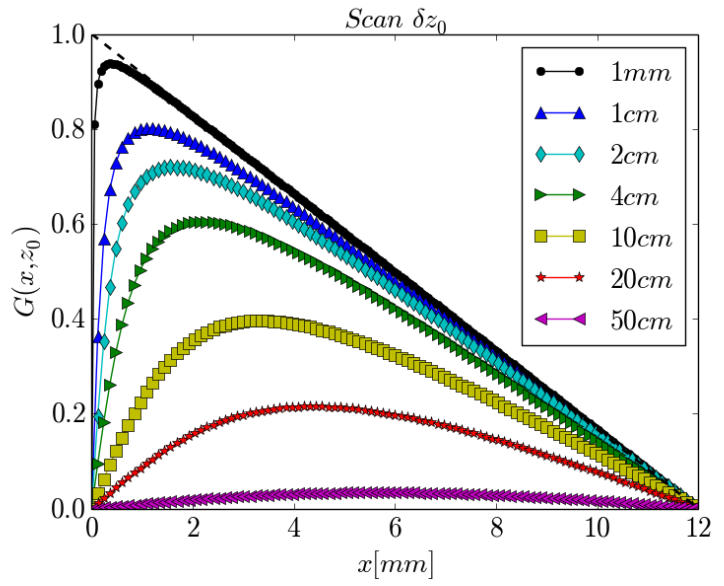

FIGURE 3. Green's function $G\left(x, z_{0}\right)$ versus radial coordinate $x$ for increasing parallel distance $\delta z_{0}=\left(L_{/ /} / 2+z_{0}\right)$ from wave emission point $z=z_{0}$ to left parallel boundary $z=-L_{/ /} / 2$.

Simulation with ASDEX-Upgrade parameters used in [10].

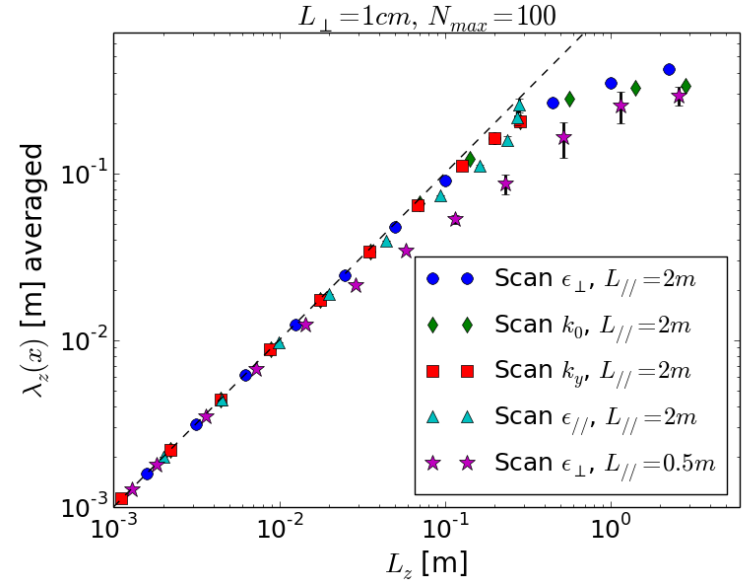

FIGURE 4. Parallel e-fold decay length $\lambda_{z}(x)$ of $G\left(x, z_{0}\right)$, fitted numerically and averaged over 20 values of $x$, versus $L_{z}$ from eq. (4), for 5 scans of the main parameters in the asymptotic model. Error bars: dispersion of $\lambda_{z}(x)$ over $x$.

As $\delta z_{0} \rightarrow 0, G\left(x, z_{0}\right)$ tends asymptotically to $G\left(x,-L_{/ /} / 2\right)=\sinh \left[k_{y}\left(L_{-}-x\right)\right] / \sinh \left[k_{y} L_{1}\right]$ (dashed curve on Fig. 3). As $\delta z_{0}$ increases, $G\left(x, z_{0}\right)$ generally decreases towards $G\left(x, L_{/ / /} / 2\right)=0$, and its radial maximum progressively moves away from $x=0$. This indicates parallel and radial proximity effects in the RF-sheath excitation. For fixed $x$, the way the Green's 
function decreases with $\delta z_{0}$ depends on the input parameters. It therefore changes dramatically when the plasma is replaced with vacuum. In a series of simulations the e-fold parallel decay length $\lambda_{z}(x)$ of $G\left(x, z_{0}\right)$ was fitted numerically for 20 values of $x$ from 0 to $L_{\text {. }}$. Figure 4 plots $\lambda_{z}(x)$ averaged over $x$, for various parametric scans. Two characteristic parallel scale-lengths were identified for the SW evanescence in formula (5): $L_{z}$ and $L_{/ /}$. For $L_{z}<<L_{/ /}$, $\lambda_{z}(x)$ weakly depends on $x$ and is approximately $L_{z}$. This can be deduced from the decay of $F$ in Fig. 2: indeed the two lateral boundaries get sufficiently far apart so that only $n=0$ and $n=-1$ matter in eq. (5). A decay length similar to $L_{z}$ was already evidenced in an earlier model of RF sheaths with prescribed width excited by evanescent SW [9]. As $L_{z} \sim L_{/ /}$, Fig. 3-4 show that $\lambda_{z}(x)$ is more scattered in $x$ but remains below $L_{z}$ and $L_{/ /}$. For $L_{z}>>L_{/ /}, G\left(x, z_{0}\right)$ approximately decreases linearly as $G\left(x,-L_{/ / /} / 2\right) \times\left(1-\delta z_{0} / L_{/ /}\right)$. The $2 \mathrm{D}$ Green's function formalism can be extended to $3 \mathrm{D}$ simulation domains, and line integral (3) is replaced by a surface integral over a $2 \mathrm{D}$ input RF field map $E_{/ / a p}(y, z)$.

\section{PRACTICAL IMPLICATIONS, LIMITATIONS AND PROSPECTS}

Within our asymptotic model, the excitation of sheath voltage RF oscillations $V_{R F}$ by the evanescent $\mathrm{SW}$ decreases with increasing parallel distance sheath/SW emission point. Typical decay lengths are always smaller than typical antenna parallel extensions $L_{/ /}$. Consequently, when the radiated $E_{/ / a p}$ map exhibits parallel anti-symmetry, an attenuation factor prevents the cancellation of the relevant integrals for $V_{R F}$ in eq. (3). Sheath oscillations therefore persist with anti-symmetric strap toroidal phasing (e.g. $[0, \pi]$ ), while the previous formula for $\tilde{V}$ predicts that $\int E_{/ / .} d l=0$ for symmetry reasons. Besides, the sheaths at the two ends of the same open field line can oscillate differently, depending on the parallel symmetry of $E_{/ / \text {ap }}$ map. Beyond line-integrated quantities, $V_{R F}$ at an IC antenna side limiter appears mainly sensitive to $E_{/ / a p}$ emission by active or passive conducting elements near this limiter, as suggested by experimental observations in ref. [2] [3]. Toroidal proximity effects could justify current attempts at reducing the RF fields induced near antenna boxes to attenuate the sheath oscillations in their vicinity [3]. Although these conclusions were reached in a rectangular box with homogeneous plasma in the "wide sheath" limit, we believe that they persist qualitatively with more complex geometry, density gradients and finite sheath widths.

The SSWICH-SW model predicts that the direct excitation of sheath oscillations by the evanescent SW is only intense in the antenna near RF field [5] [11] and loses efficiency above a parallel distance of the order of $L_{z}$. However, RF-induced DC biasing of the SOL plasma has often been observed experimentally at parallel distances far larger than $L_{z}$ [2], [3], [12-15]. To interpret these measurements, extra physical mechanisms not discussed in the present paper need to be added. In very tenuous SOLs below the lower hybrid resonance $\left(\varepsilon_{ \pm}=0\right)$, the SW becomes propagative [16] and can possibly excite RF sheaths at large parallel distances [17]. At higher densities, the Fast Wave becomes propagative. It can excite so-called "far-field RF-sheaths" if $\mathbf{B}_{\mathbf{0}}$ is not strictly normal to the walls [18]. In the absence of propagating RF waves, ref. [5] showed that the transport of DC current can still spread a DC bias to remote areas from the near-field regions where SW direct sheath excitation is efficient. Within a European project outlined in ref. [11], work is ongoing to include these extra physical mechanisms into more realistic models of coupled RF wave propagation and DC plasma biasing. This implies, inter alia, moving towards 3D full-wave propagation and shaped walls [19]. Comparison with plasma measurements [3,5,10] is also key for code validation.

Acknowledgements. This work has been carried out within the framework of the EUROfusion Consortium and has received funding from the Euratom research and training programme 2014-2018 under grant agreement No 633053. The views and opinions expressed herein do not necessarily reflect those of the European Commission.

\section{REFERENCES}

1. Perkins F.W., Nucl. Fus. 29 (4) 1989, p. 583

2. L. Colas et al., JNM 438 (2013) S330-S333

3. V. Bobkov et al., these proceedings (2015)

4. L. Colas et al., PoP 19, 092505 (2012)

5. J. Jacquot et al. PoP 21, 061509 (2014)

6. T.H. Stix, "Waves in Plasmas", AIP Press 1992

7. D.A. D'Ippolito \& J.R. Myra, PoP 13102508 (2006)

8. H. Kohno et al. $C P C \mathbf{1 8 3}$ (2012) p. 2116

9. J. R. Myra \& D. A. D'Ippolito, PPCF 52015003 (2010)
10. Křivská et al., these proceedings (2015)

11. L. Colas et al., Proc. $21^{\text {st }}$ IAEA FEC conference, $\mathrm{S}^{\mathrm{t}}$ Petersburg (Russia) 2014, TH/P6-9

12. Cziegler et al., PPCF 54 (2012) 105019

13. C. C. Klepper et al. JNM 438 (2013) S594

14. M. Kubič, et al., JNM 438 (2013) S509

15. R. Ochoukov et al. JNM 438 (2013) S875

16. L. Lu et al., these proceedings (2015)

17. Myra J.R., D’Ippolito D.A. 2008 PRL 101195004

18. D.A. D'Ippolito et al., PoP 15, 102501 (2008)

19. J. Jacquot et al., these proceedings (2015) 